Article

\title{
Effect of a Traveling Magnetic Field on Micropore Formation in Al-Cu Alloys
}

\author{
Yanjin Xu ${ }^{1, *}$, Lijun Wei ${ }^{2}$, Baoshuai Han ${ }^{1}$, Enyu Guo ${ }^{3, *}$, Mingyue Wang ${ }^{2}$ and Yanqing Su 4 \\ 1 AVIC Manufacturing Technology Institute, Beijing 100024, China; hbshit@126.com \\ 2 School of Material Science and Engineering, Beihang University, Beijing 100191, China; \\ bhwlj@buaa.edu.cn (L.W.); bhwangmy@163.com (M.W.) \\ 3 Key Laboratory of Solidification Control and Digital Preparation Technology (Liaoning Province), \\ School of Materials Science and Engineering, Dalian University of Technology, Dalian 116024, China \\ 4 School of Materials Science and Engineering, Harbin Institute of Technology, Harbin 150001, China; \\ suyq@hit.edu.cn \\ * Correspondence: xuyj_avic@aliyun.com (Y.X.); eyguo@dlut.edu.cn (E.G.); \\ Tel.: +86-10-8570-1653 (Y.X.); +86-411-8470-9500 (E.G.)
}

Received: 14 May 2018; Accepted: 5 June 2018; Published: 12 June 2018

\begin{abstract}
The effect of traveling magnetic fields (TMFs) on the grain and micro-pore formation in an $\mathrm{Al}$ alloy was studied by scanning electron microscope and X-ray microtomography in this work. The results show that with the increasing magnetic flux density, the three-dimensional morphology of the micro-pores transformed from dendrite to a relatively equiaxed structure. Quantified results show that both the micro-pore volume fraction and the average grain size of the primary phase decreased as the TMF density increased. The analyses show that the forced convection induced by TMF can break the dendrites, refine the grain size, and promote the liquid feeding, leading to the decrease in the volume fraction of the porosity and improved mechanical property. The TMF performed at different stages during solidification reveal that the maximum effect of TMF on reducing the micro-pore formation was found when TMF was applied in the stage of nucleation and the early stage of grain growth during solidification.
\end{abstract}

Keywords: aluminum alloy; solidification; X-ray tomography; porosity defects; magnetic field treatment

\section{Introduction}

The shape casting process offers an effective way to produce complex components in one single production step [1]. However, the use of this technique is also limited by the formation of defects such as micro-pore, hot tearing [2,3], and so forth. Porosity/micro-pore is one of the major defects in castings, which is usually induced by gas segregation and solidification shrinkage in the mushy zone. According to the research of Campbell [4], the presence of micro-pore reduces the mechanical properties of the cast component, including fatigue life, tensile strength, ductility, and surface quality.

No porosity could be found in the castings if the gas is absent and the feeding is adequate. However, many regions of the castings are not fed, and then the micro-pore may form in a number of ways [4]. The problem of micro-pore formation in casting alloys continues to be of interest despite the many computational models that have been proposed. The problem of the micro-pore formation is very complex, as it involves many materials (that is, initial gas content, melt purity) and processing (that is, temperature, temperature gradient, cooling rate, applied pressure) parameter interactions in complex physics [5]. It has been shown that the nucleation of the microporosity is influenced by foreign impurities [6]. According to the previous work performed by Felberbaum [7], we know that the porosity which is constrained to grow between narrow inter-dendritic liquid channels has a higher 
curvature, and thus a higher internal pressure than that of a free-growth spherical one, and the fraction of the porosity, hence, decreases with an increasing curvature. Therefore, increasing the curvature of the porosity and decreasing the grain size are effective methods to decrease the fraction of the porosity.

The introduction of the electromagnetic technique as a new method for tailoring the microstructure and micro defection of alloys has attracted increasing attention. The application of the high magnetic field suggested a possibility of adjusting the morphology of solid-liquid phase during the solidification of Al-Cu alloys [8], which shows a potential way to control the distribution and amount of porosities. Previous research [1,2] found that the application of the magnetic field can cause grain refinement, in which some of the resulting microstructures are much better than those used by other solidification technology, for example, supergravity solidification [9]. Zuo et al. [10] pointed out that the increasing external magnetic field can tilt the growth direction of the lamellar eutectic and decrease the coarse eutectic lamellar spacing during solidification, leading to a higher strength. The work by Erb et al. [11] indicated that even a low magnetic field ( 1 to 10 millitesl as) has a significant effect on the orientation and distribution of the reinforced particles in artificial composites. Therefore, the application of a magnetic field during the solidification process is an effective method to reduce defects and optimize microstructure and properties. However, despite the successful applications of the magnetic field in the material fabrication, the mechanisms of the effect of the electromagnetism on the melt are not yet well understood. Therefore, more work is needed to understand the role of the magnetic field in each stage of the solidification progress.

The recent development of high-resolution X-ray tomography imaging techniques proposed a useful method to explore the structure evolution in the solidification process. Holm et al. [12] have enabled three-dimensional (3D) observations of the microporosity morphology. Lee and Hunt [13] first applied this technique to visualize the formation of the porosity in Al-Cu alloys with a micro-focus X-ray source. However, the resolution was about $25 \mu \mathrm{m}$ and the intensity of the beam was also a limiting factor. The synchrotron X-ray tomography provides higher resolution and higher flux capabilities at beamlines, and, thus, better characterization can be performed, which results in an improved understanding of the mechanisms involved in the material processing [14].

In this work, the effect of TMF on the micro-pore formation and on the morphology of micro-pores and grain size are compared in the solidification process of $\mathrm{Al}-\mathrm{Cu}$ alloys under a traveling magnetic field (TMF) with various magnetic flux densities. X-ray tomography was performed to characterize the 3D morphology of the micro-pores in Al-Cu alloys after the alloy was solidified. Meanwhile, different strategies of magnetic treatments were performed in different stages of solidification to evaluate the effect of this treatment, and its mechanism was discussed and revealed.

\section{Materials and Methods}

The schematic illustration of the experimental apparatus is shown in Figure 1. The traveling magnetic field was created by means of applying out-of-phase alternating current (AC) currents (50 Hz) to six coils with a star arrangement connected vertically one upon the other. As a result, a downward meridional traveling Lorentz magnetic field was induced in the sample melt. This technique has already been detailed in previous work [15]. A nonmagnetic stainless-steel frame was designed and assembled to hold the sample firmly. The sample had a diameter of $3.5 \mathrm{~mm}$ and a length of $100 \mathrm{~mm}$ and was placed in a quartz tube fixed in the center of the cavity. The nonmagnetic stainless-steel frame, which allowed water to stream in and out, was installed and thus enabled the equipment to control the cooling rate by adjusting the flow rate of the streaming water. An electric heating furnace was used to heat and melt the sample. The furnace was removed through the opening of the upper cylinder when the TMF generator was applied.

The $\mathrm{Al}$ alloy studied in this work had a composition of $3.8 \mathrm{Cu}-0.4 \mathrm{Mn}-0.3 \mathrm{Ti}-0.1 \mathrm{Cd}-0.25 \mathrm{~V}-\mathrm{Al}$ (bal. in wt. \%). The sample was placed in the center of the TMF generator and the heating furnace was set around the sample and turned on to heat and melt the sample up to $1023 \mathrm{~K}$. The molten sample was kept at this temperature for $10 \mathrm{~min}$ before the furnace was removed. Then the TMF generator 
was applied at different stages of the cooling process to the ambient temperature. The magnetic flux densities of the TMF were set as $24 \mathrm{mT}, 32 \mathrm{mT}$, and $48 \mathrm{mT}$, respectively. For comparison, a sample was also prepared without TMF application.

The microstructures of the as-cast samples were examined by scanning electron microscopy (SEM) (Quanta 200FEG, FEI, Hillsborough, OR, USA) in the cross-section of the sample and 3D X-ray microtomography. The X-ray microtomography was carried out at the BL13W1 beamline in the Shanghai Synchrotron Radiation Facility (SSRF). An X-ray energy of $30 \mathrm{keV}$ was used to penetrate a cylindrical sample of $3.5 \mathrm{~mm}$ in diameter and $2-5 \mathrm{~mm}$ in height with a voxel size of $3.7 \mu \mathrm{m}$. A $2048 \times 2048$ pixel CoolSnap K4 CCD camera (Coolsnap, Photometrics, Tucson, AZ, USA), coupled with a $\mathrm{CdWO}_{4}$ scintillator screen, was used to record the projections. Each 3D data set contains 1440 projections that were collected with an exposure time of $1 \mathrm{~s}$. The projections were reconstructed using a filtered-back-projection algorithm. The raw images were preprocessed in Image J (NIH, Bethesda, MD, USA) and then further analyzed in Avizo (VSG Group, Bordeaus, France). A median filter was applied in Avizo to reduce the noise of the image. The tensile tests were conducted on an electronic universal mechanical testing machine (Instron5569, Instron Co., Canton, $\mathrm{OH}, \mathrm{USA}$ ) at an initial strain rate of $5 \times 10^{-2}$. The length of the sample gauge was $20 \mathrm{~mm}$.

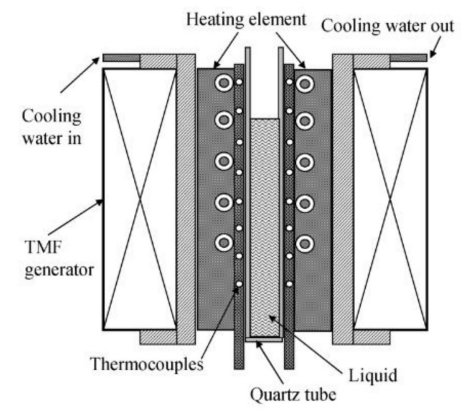

Figure 1. The schematic illustration of the experimental apparatus.

\section{Results and Discussion}

In order to determine the effect of magnetic flux density on the volume fraction and morphology of the micro-pores, Al-alloy samples were solidified in the TMF with various magnetic flux densities. Figure 2 shows the typical microstructures formed without TMF treatment (Figure 2a) and with $48 \mathrm{mT}$ TMF treatment (Figure $2 \mathrm{~b}$ ). The microstructure was composed of primary dendrites and white $\mathrm{Al}^{-\mathrm{Al}_{2} \mathrm{Cu}}$ eutectic phase. It is evident that micro-pores (dark regions) were present in the interstices between the primary dendrites in the sample without TMF treatment, and shrinkage porosity appears as isolated holes, both of which indicated that feeding without TMF treatment in the densely packed dendrite arms was very difficult. For comparison, the micro-pores were inhibited significantly after TMF treatment, as shown in Figure 2b, suggesting that the TMF has a significant effect on reducing the porosity defects.
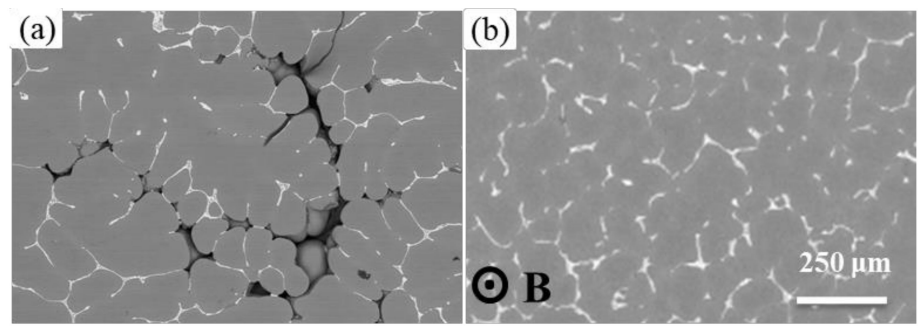

Figure 2. The SEM images showing microstructures in the as-cast $\mathrm{Al}$ alloys: (a) without and (b) with $48 \mathrm{mT}$ TMF treatment. Shrinkage porosities (dark regions) are observed in the Al-alloy without TMF treatment. The direction of the TMF is indicated in $(\mathbf{b})$ by point $\mathrm{B}$, represents from inward to outside. Scales bar is the same for $(\mathbf{a}, \mathbf{b})$. 
The 3D shapes of the typical micro-pores formed in $\mathrm{Al}$ alloys treated under various magnetic flux densities are shown in Figure 3. Without TMF treatment, shown in Figure 3a, the micro-pores have an elongated and highly-connected shape along the thermal gradient direction (horizontal). However, with the application of TMF, the morphologies of the micro-pores are more likely to be equiaxed, and the distribution of the micro-pores has been changed significantly. Meanwhile, the size of the micro-pores tends to decrease gradually with the increase of the magnetic flux density.

A schematic showing the effect of TMF on the distribution and morphology of the micro-pores is presented in Figure 3e. Due to the low copper content, the volume fraction of the primary phase was very high $(\sim 90 \%)$ in the solidification microstructure. When the volume fraction of the primary phase is low in the early solidification progress, a significant amount of fluid can flow through the dendrite network and feed the solidification shrinkage. As the solidification progress continues, the dendrite would grow and interconnect to form a complex extended network in the three-dimensional space, as can be seen in Figure 3. In this situation, the micro-pores have to nucleate in the mushy region after the formation of the primary aluminum dendrites, and then grew by replacing and pushing away the surrounding liquid. As the pushing force induced by the pore was not high enough to break the primary phase, the solid phase grows along or even into the micro-pores. As a result, the micro-pores formed in this way had a very complex morphology. In the case of the alloy in this work, the solidification temperature range is $\sim 120^{\circ} \mathrm{C}$, and the fraction of the eutectic microstructure is relatively small. This makes the feeding very difficult for the rest of the liquid to solidify, which usually results in high levels of micro-pores. Since the micro-pores have formed in the mushy zone after the solidification of the primary phase "skeleton", the shape of the micro-pores is determined by the morphology of the primary phase accordingly. It was observed that micro-pores forming near the dendritic phase were also dendritic in structure.
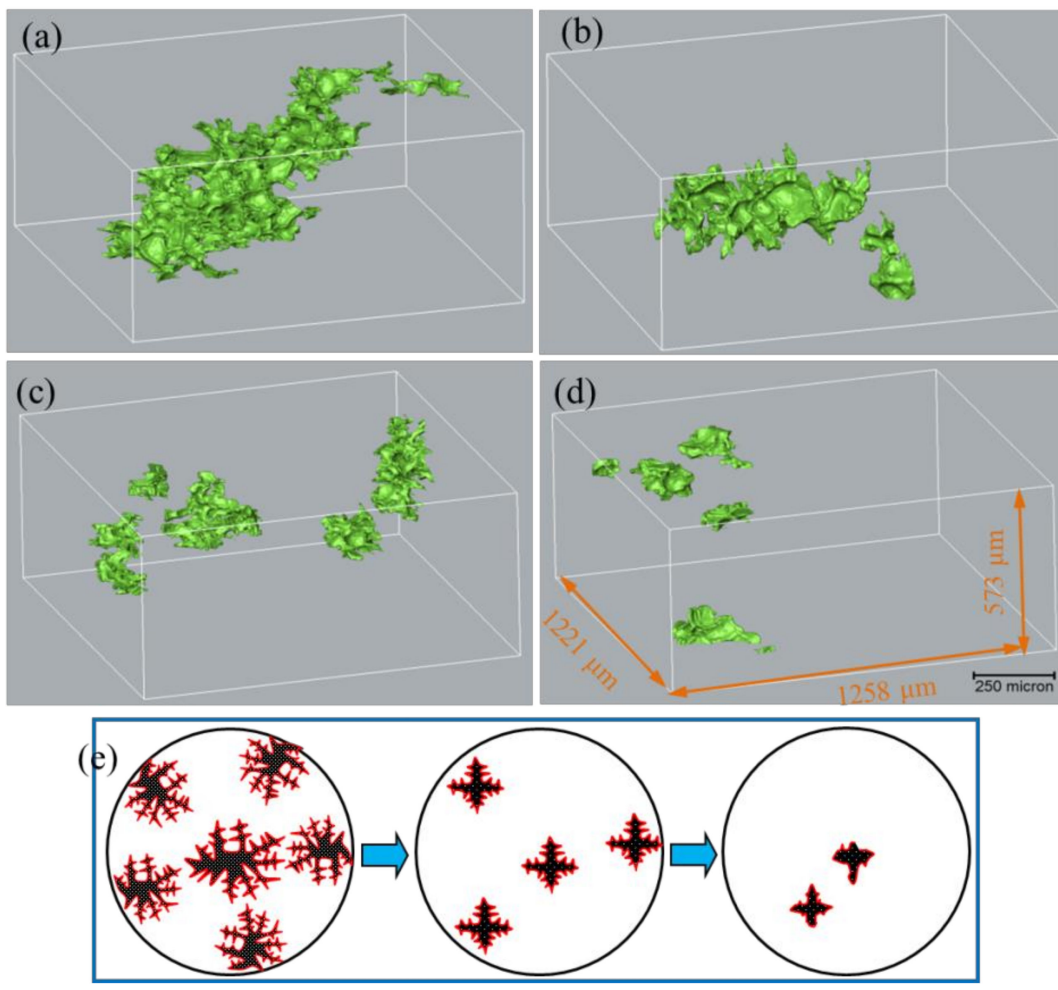

Figure 3. The 3D morphology of the micro-pores in the Al-Cu alloys under TMF with a magnetic flux density of (a) $0 \mathrm{mT}$ (b) $24 \mathrm{mT}$ (c) $32 \mathrm{mT}$ (d) $48 \mathrm{mT}$. (e) is the schematic showing the effect of TMF on the distribution and morphology of the micro-pores. 
In order to better understand and evaluate the contribution of TMF, a special study was designed to characterize and establish the relationship between the quantified micro-pore fraction and grain size under various experimental conditions, and the result is shown in Figure 4. It is found that the volume fraction of micro-pore decreases gradually with the increasing TMF intensity and decreasing grain size. In the case of our study, TMF plays important roles in two ways: one is that it refines the grains and the other one is that it enhances the feeding pressure $[16,17]$. In theory, grain refinement can promote mass feeding [3]. Practically, when the solid fraction exceeds a threshold value, the dendrites start to become a coherent network and the free grains are so large that only smaller ones can pass through the network, thus, leaving the large grains pinned in the solid skeleton and the feeding path blocked prematurely. When TMF is introduced, the solid skeleton is likely to be broken up by the imposed Lorentz force during solidification, and the grains are refined [18]. Moreover, due to the stirring effects of the TMF, the distribution of solutes becomes more uniform, which are helpful to precipitate equiaxed grains instead of the solid skeleton [19]. Therefore, the slurry of the liquid metal can move to the front of the solidification interface sufficiently, increasing feeding ability and decreasing the volume fraction. Thus, it is assumed that the suppression of the shrinkage porosity under TMF was mainly caused by different aspects including the grain refinement, the enhanced feeding pressure induced by TMF and a combined effect of them. With the increase of the TMF intensity, the grains were refined gradually and became more equiaxed, making the micro-pores transform from dendrite to equiaxed structure accordingly, as shown in Figure $3 b-d$. These results indicate that the TMF can change the micro-pore morphology, and this change is mainly caused by the shape of the primary solid skeleton. Although many research reports $[14,18,20]$ have studied the grain refining under the magnetic field, the relationship between the grain refining and porosity has not been revealed. In this work, we found that refining the grain size can improve the feeding ability in the solidification progress and decrease the volume fraction of the porosity under TMF, suggesting a promising process to improve the quality of the casting Al-alloy.
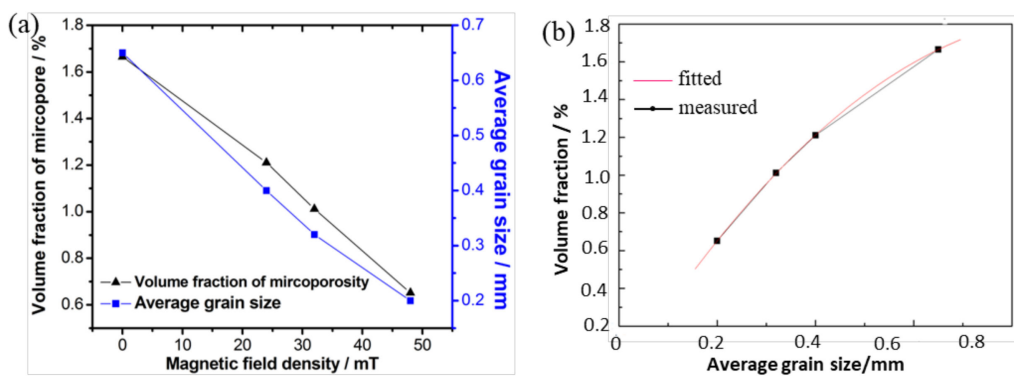

Figure 4. (a) The effect of TMF on the micro-pore fraction and grain size in Al-alloy solidified under different magnetic flux conditions; (b) result showing the relationship between the volume fraction of the micro-pore and the average primary grain size.

The cooling curves were recorded during all the experiments and the influence of TMF on the cooling curve was found to be insignificant. Thus, the TMF feeding mechanism is discussed based on its effect on different stages. In order to elucidate the feeding mechanism of the shrinkage porosity formation of this material under TMF, nine treatment strategies under the TMF intensity of $48 \mathrm{mT}$ were carried out according to the cooling curve of the specimen solidified without the TMF, as shown in Figure 5a. The relationship between the volume fraction of micro-pores and the grain size with a given TMF treatment at different solidification stages is presented in Figure $5 b$, which indicates the specific stage where the TMF played the main role. The TMF treatment at Stage 1 and 2, where the alloy was in a complete liquid status, shows no effect on either grain refinement or micro-pore fraction. This indicates that the TMF has no inoculation effect on the liquid Al alloy. When the TMF was applied in the nucleation Stage 3 (before crystal growth had started), both the grain size and the volume fraction of the micro-pores was reduced, as compared with those in Stage 1 and 2, suggesting that the 
TMF can refine grains and suppress the shrinkage porosity by imposing effects on the nucleation stage. Our results are in agreement with the report by Liao et al. [21]. The results also show that the TMF in Stage 3 only affected the grain nucleation partly, while the TMF applied in the whole nucleation stage and the early stage of the grain growth, that is, Stage 4 and 5 respectively, can further play an important role in reducing the grain size. Both the strategies led the similar results, yielding refined grains and suppressed shrinkage porosity. Moreover, both the volume fraction of the micro-pores and the average grain size are minimal with the TMF treated at these two stages. At Stage 6, TMF was introduced within the middle crystal growth stage, wherein micro-pores were suppressed slightly due to grain refinement. It can also be seen from Figure $5 b$ that the application of the TMF at the last stage of crystal growth almost has no effect on either the grain size or the micro-pore volume fraction (Stages 7 and 8). Interestingly, when applying the TMF for the entire solidification process (Stage 9), the grains were refined greatly and the volume fraction of the micro-pores was also significantly reduced, similar to that observed in Stage 4 and 5. Therefore, it is concluded that the defect of the pore formation and the mechanism of grain refinement are closely related to the nucleation process and that, in order to maximize the grain refinement effect, TMF should be applied in Stage 4 and 5 or the whole process stage. Consider the resources, that is, the time, efforts, and the instrument time for the TMF generation, applying TMF at Stages 4 and 5 should be enough instead of applying it for all the stages.
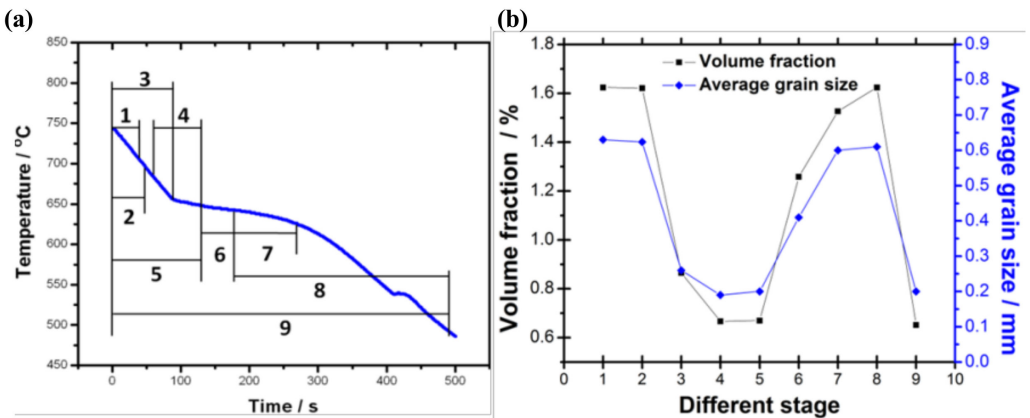

Figure 5. The effect of TMF on the micro-pore fraction and grain size in Al-alloy solidified under different conditions: (a) schematic sketch of solidification stages treated with TMF; (b) evolution of volume fraction and average grain size in different solidification stages with TMF treatment.

The aforementioned experimental results clearly show that grain refinement by TMF is primarily achieved during the nucleation stage and the early stage of the crystal growth other than in the stages when only the liquid phase exists or in the last stage of crystal growth. Shrinkage porosity suppression, accompanied by grain refinement, is achieved at the same stage under TMF application. Compared with the two curves in Figure 5b, it is obvious that the change of the volume fraction of the micro-pores exhibits the same trend as the grain size, which supports our hypothesis that grain refinement is helpful to suppress the porosity. According to the research of Fleming [22], we know that the shear strength of the solid phase can be neglected when the volume fraction of the solid phase is below 0.2 and that this shear strength increases with the increase of the solid phase volume fraction when the fraction is larger than 0.2. Therefore, the forced convection induced by TMF can break the small dendrites easily in the nucleation progress, that is, Stage 3, 4, and 5, which leads to a great refining. However, the shear strength of the solid phase increased dramatically after the fraction of the solid phase reached 0.4 . In our study, the electromagnetic force induced by TMF is relatively low. It is believed that the forced convection induced by TMF cannot break the dendrites in this situation. This might account for the observation that TMF cannot refine the grains in the last stage of crystal growth. In the last crystal growth stage, although some residual liquid still existed in the interdendritic zone, the micro-pore formation cannot be suppressed even with TMF. This suggests that the enhanced electromagnetic pressure has no effect on the shrinkage porosities. As a result, the most appropriate stage to apply the TMF is in the nucleation stage and the early stage of the grain growth when the 
solid phase is below 0.2 , in which the TMF can refine the grain to the greatest extent in this process and suppress the porosity in the solidification.

Due to the decreased micro-pores, the mechanical properties of the alloys under TMF were enhanced and the tensile results are shown in Figure 6. For example, the tensile strength and elongation of the alloy increased with the increasing TMF intensity. The optimal mechanical properties (strength of $214 \mathrm{MPa}$, elongation of $11.3 \%$, increased by $8.0 \%$ and $29.9 \%$, respectively, as compared with the samples without TMF treatment) were obtained in the $\mathrm{Al}$ alloy treated with $48 \mathrm{mT}$ TMF. Under a tensile loading condition, cracks would be initiated from the weak position, that is, micro-pores, resulting in an early failure of the sample when a number of pores are present in the material. The small grains present in the materials also leads to a greater strength and better elongation. This also suggests that the effect of TMF has a positive effect on improving the mechanical properties. Therefore, TMF treating is a promising method to improve the microstructure and mechanical properties of the casting $\mathrm{Al}$ alloys.

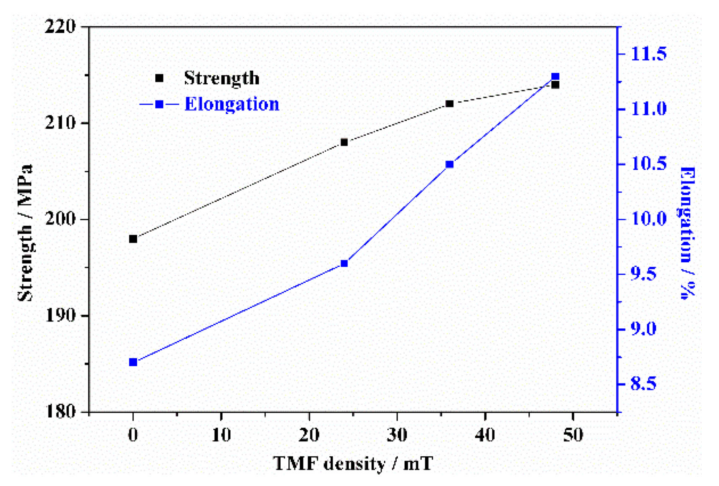

Figure 6. The tensile strength and elongation of Al-alloys solidified under TMF.

\section{Conclusions}

In summary, we studied the effect of a magnetic field on the micro-pore formation in an $\mathrm{Al}$ alloy. It is found that TMF treatment can suppress the shrinkage porosity effectively, resulting in a reduction in both volume fraction of the micro-pore and grain size after TMF treatment. The analyses show that TMF can enhance the feeding ability in the solidification progress, resulting in decreased volume fraction of the porosity in the $\mathrm{Al}$ alloy. The grain refinement can also be introduced by the TMF. Detailed mechanism investigations revealed that micro-pores can only be suppressed by the TMF when it is applied during nucleation and the early stage of the grain growth. Therefore, it is suggested that the TMF be applied in the nucleation stage instead of the whole solidification progress. The refined grains and reduced micro-pores caused by the TMF in the materials lead to improved mechanical properties.

Author Contributions: Y.X. guided the investigation and experiments, analyzed the experiment data and mechanism, wrote the paper, and revised the paper; L.W. optimized the research plan and revised the paper; B.H. made the diagram and graph, and revised the paper; E.G. made the diagram and graph, and revised the paper; M.W. collected the data, and revised the paper; Y.S. searched literature, and optimized the diagram and charts.

Funding: This research was funded by the Defense Industrial Technology Development Program (Grant No. JCKY2017205B032).

Acknowledgments: The use of X-ray synchrotron beam line BL13W1 at the Shanghai Synchrotron Radiation Facility (SSRF) is acknowledged. The authors wish to express thanks to them. Helpful discussion with James Mertens in the Arizona State University is also greatly appreciated.

Conflicts of Interest: The authors declare no conflict of interest. 


\section{References}

1. Meidani, H.; Desbiolles, J.L.; Jacot, A.; Rappaz, M. Three-dimensional phase-field simulation of micropore formation during solidification: Morphological analysis and pinching effect. Acta Mater. 2012, 60, 2518-2527. [CrossRef]

2. Dantzig, J.A.; Rappaz, M. Solidification; EPFL: Lausanne, Switzerland, 2009; pp. 479-500.

3. Liotti, E.; Lui, A.; Vincent, R.; Kumar, S.; Guo, Z.; Connolley, T.; Dolbnya, I.P.; Hart, M.; Arnberg, L.; Mathiesen, R.H. A synchrotron X-ray radiography study of dendrite fragmentation induced by a pulsed electromagnetic field in an Al-15Cu alloy. Acta Mater. 2014, 70, 228-239. [CrossRef]

4. Campbell, J. Castings; Butterworth-Heinemann: London, UK, 2003; pp. 280-396.

5. Stefanescu, D.M.; Catalina, A.V. Physics of microporosity formation in casting alloys-Sensitivity analysis for Al-Si alloys. Int. J. Cast Met. Res. 2011, 24, 144-150. [CrossRef]

6. Roy, N.; Samuel, A.M.; Samuel, F.H. Porosity formation in Al-9 Wt pct Si-3 Wt pct Cu alloy systems: Metallographic observations. Metall. Mater. Trans. A 1996, 27, 415-429. [CrossRef]

7. Felberbaum, M.; Rappaz, M. Curvature of micropores in Al-Cu alloys: An X-ray tomography study. Acta Mater. 2011, 59, 6849-6860. [CrossRef]

8. Li, X.; Fautrelle, Y.; Ren, Z. Influence of an axial high magnetic field on the liquid-solid transformation in Al-Cu hypoeutectic alloys and on the microstructure of the solid. Acta Mater. 2007, 55, 1377-1386. [CrossRef]

9. Yang, Y.; Song, B.; Yang, Z.; Song, G.; Cai, Z.; Guo, Z. The refining mechanism of super gravity on the solidification structure of al-cu alloys. Materials 2016, 9, 1001. [CrossRef] [PubMed]

10. Zuo, X.; Zhao, C.; Zhang, L.; Wang, E. Influence of growth rate and magnetic field on microstructure and properties of directionally solidified Ag-Cu eutectic alloy. Materials 2016, 9, 569. [CrossRef] [PubMed]

11. Erb, R.M.; Libanori, R.; Rothfuchs, N.; Studart, A.R. Composites reinforced in three dimensions by using low magnetic fields. Science 2012, 335, 199-204. [CrossRef] [PubMed]

12. Holm, E.A.; Duxbury, P.M. Three-dimensional materials science. Scr. Mater. 2006, 54, 1035-1040. [CrossRef]

13. Lee, P.D.; Hunt, J.D. Hydrogen porosity in directional solidified aluminium-copper alloys: In situ observation. Acta Mater. 1997, 45, 4155-4169. [CrossRef]

14. Zhang, Y.; Song, C.; Zhu, L.; Zheng, H.; Zhong, H.; Han, Q.; Zhai, Q. Influence of electric-current pulse treatment on the formation of regular eutectic morphology in an Al-Si eutectic alloy. Metall. Mater. Trans. $B$ 2011, 42, 604-611. [CrossRef]

15. Su, Y.; Xu, Y.; Zhao, L.; Guo, J.; Fu, H. Effect of electromagnetic force on melt induced by traveling magnetic field. Trans. Nonferrous Met. Soc. China 2010, 20, 662-667. [CrossRef]

16. Xu, Y.; Su, Y.; Luo, L.; Liu, J.; Guo, J.; Fu, H. Study on in situ Al-Si functionally graded materials produced by traveling magnetic field. Sci. Eng. Compos. Mater. 2012, 19, 209-214. [CrossRef]

17. Su, Y.; Xu, Y.; Luo, L.; Zhang, T.; Wu, S.; Guo, J. Study on traveling magnetic field casting of sheet components. China Foundry 2010, 7, 259-264.

18. Zhang, Y.; Cheng, X.; Zhong, H.; Xu, Z.; Li, L.; Gong, Y.; Miao, X.; Song, C.; Zhai, Q. Comparative study on the grain refinement of Al-Si alloy solidified under the impact of pulsed electric current and travelling magnetic field. Metals 2016, 6, 170. [CrossRef]

19. Zhang, C.; Zhong, H.G.; Wu, C.S.; Ni, J.; Zhai, Q.J. Effect of cooling rate and forced convection on as-cast structure of 2205 duplex stainless steel. China Foundry 2015, 12, 32-38.

20. Metan, V.; Eigenfeld, K.; Räbiger, D.; Leonhardt, M.; Eckert, S. Grain size control in Al-Si alloys by grain refinement and electromagnetic stirring. J. Alloy Compd. 2009, 487, 163-172. [CrossRef]

21. Liao, X.; Zhai, Q.; Luo, J.; Chen, W.; Gong, Y. Refining mechanism of the electric current pulse on the solidification structure of pure aluminum. Acta Mater. 2007, 55, 3103-3109. [CrossRef]

22. Flemings, M.C. Behavior of metal alloys in the semi-solid. Mater. Trans. A 1991, 22, 957-981. [CrossRef]

(C) 2018 by the authors. Licensee MDPI, Basel, Switzerland. This article is an open access article distributed under the terms and conditions of the Creative Commons Attribution (CC BY) license (http:/ / creativecommons.org/licenses/by/4.0/). 